\title{
ORBITALS IN GENERAL CHEMISTRY, PART I: THE GREAT DEBATE
}

\author{
Guy Lamoureux ${ }^{\mathrm{a}, \mathrm{b}, *,(\mathbb{D}}$ and John F. Ogilvie ${ }^{\mathrm{a}, \mathrm{c}, \mathrm{d}}$ \\ aUniversidad de Costa Rica, Escuela de Química, 11501-2060 San Pedro de Montes de Oca, San José, Costa Rica \\ ${ }^{\mathrm{b}}$ Centro de Investigaciones en Productos Naturales (CIPRONA), 11501-2060 San Pedro de Montes de Oca, San José, Costa Rica \\ ${ }^{\circ}$ Centre for Experimental and Constructive Mathematics, Department of Mathematics, Simon Fraser University, 8888 University \\ Drive, Burnaby, British Columbia V5A 1S6 Canada \\ Institute of Quantum Physics, Irkutsk National Research Technical University, 83 Lermontov Street, Irkutsk 664074, Russian \\ Federation
}

Recebido em 14/06/2020; aceito em 13/08/2020; publicado na web em 08/10/2020

\begin{abstract}
In Part I of a three-part series, we present an objective survey of the technical literature discussing the inclusion of orbitals in highschool and post-secondary general chemistry. We start with the definition of orbitals. The presentation of orbitals in secondary (high) school is presented with arguments for and against. The content of orbitals in the curriculum of general chemistry is presented and summarized in terms of 1) electron configuration, 2) periodic table, 3) molecular structure and 4) reactivity. Previous discussion in favor of and against each theme is presented. Recent research in chemical education is also reviewed.
\end{abstract}

Keywords: teaching general chemistry; orbitals; quantum mechanics; molecular structure.

\section{INTRODUCTION}

"In commencing the study of a physical science, we ought to advance no idea but what is a necessary consequence, and immediate effect, of an experiment or observation." Lavoisier $^{1}$

According to a reputable textbook ${ }^{2}$ for general chemistry, "the solutions to Schroedinger's equation for the hydrogen atom yield a set of wave functions called orbitals". This definition clearly emphasizes that orbitals are simply algebraic formulae.

Mulliken invented the term orbital, defined with characteristic obfuscation as "something as much like an orbit as is possible in quantum mechanics."3 Practicing chemists have not restricted themselves to either definition, but have extended it to mean a wave function as a state, or a basis state or basis wave function, or some mathematical ansatz that is used to construct a wave function, or even some region of space in an atomic environment. Chemists thus pepper their papers with orbitals in such contexts as 'atomic orbitals (AO)', 'canonical Hartree-Fock orbitals', 'localized molecular orbitals (LMO)', 'valence bond orbitals (VBO)', 'hybrid atomic orbitals (HAO)', 'spin-orbitals', and 'Kohn-Sham orbitals (KSO)'. ${ }^{4}$ 'Dyson orbitals', confusingly, are not orbitals at all. ${ }^{5}$ Whatever definition of an orbital one uses, the indistinguishability of electrons must invariably apply, implying an inability to observe these mathematical constructs. "It is impossible to claim that a given electron occupies a specific orbital...It follows that the orbitals cannot be considered as unique in systems with two or more electrons, and this non-uniqueness of the orbitals precludes their experimental observation." ${ }^{4}$

Most students seem not to be limited by these definitions. For example, when students in general chemistry are examined, they confuse 'atomic orbitals' with 'molecular orbitals' and 'orbitals' with 'orbits' (based on a planetary model of the atom). ${ }^{6,7}$ A common complaint is that, as there is insufficient time to understand the orbital model, these misconceptions block new learning, so becoming an impediment to understanding. ${ }^{8}$ Part of the problem might be the

*e-mail: guy.lamoureux@ucr.ac.cr polysemy of the word "orbital' ${ }^{\text {}}$ - this word conveys various meanings, both scientific and emotional, to a particular person. Discussion about the teaching of such a confused idea follows.

There is perhaps no teaching concept more disputed, nor more amenable to extreme opinion, than the presentation of orbitals in general chemistry. Before 1957, most textbooks for introductory chemistry (except Pauling's textbook) included not 'Schroedinger' nor 'orbital' nor 'hybridization'. ' This lack of orbital models soon altered, such that at the end of the century all textbooks based $20 \%$ of their coverage on topics nominally derived from quantum mechanics. The inclusion of orbitals has not lacked outspoken antagonists; these voices against the teaching of orbitals in introductory chemistry seem to have been lost or ignored. Astonishingly, we could find no reference after 1997 discussing whether or not to exclude orbitals in the curriculum for general chemistry.

Part of the reality is that "...implicitly, many (most?) textbook authors and curriculum programmers favor the use of orbitals to introduce chemical theory, as they include the issue not only in courses of college chemistry, but even in the (upper) secondary education in many countries. In our opinion, this acritical acceptance of the teaching of orbitals is just another consequence of the widespread ignorance of the complex status of quantum jargon within FMT [Folk Molecular Theory]. We think that for many chemistry teachers, and this is of course just a feeling with no empirical support, orbitals are simply necessary for the quantum [QC] description of a microscopic system. In this vision, there is no QC without orbitals."

Our interest in the presentation of chemistry concepts in general chemistry requires a complete review of how we arrived at this situation in year 2020. It is time to reopen the debate about what to include in the curriculum and to weigh critically all evidence in favor or against.

\section{DISCUSSION}

\section{Teaching of orbitals in secondary (high) school}

In 1979, Morwick defended the teaching of orbitals in secondary school for two reasons. ${ }^{10}$ First, the abstract concepts of orbitals and 
electron configuration are "indispensable intellectual scaffolding" of further, more advanced topics. Morwick claimed that the language and images of orbitals should be taught in secondary school, anticipating mathematical quantum theory at the university level. Students in secondary school should be taught the shape of the orbitals $(s, p, d, \ldots)$ and their energy levels $(1 s<2 s<2 p \ldots)$, implicitly assuming spherical polar coordinates, leaving hybridization and antibonding orbitals as optional depending on the teacher. Second, as quantum chemistry is a "fundamental aspect" of chemistry; it should be introduced in secondary school with the other fundamentals. ${ }^{10}$

Whether orbitals are "indispensable" or "fundamental" has been seriously questioned. Various authors have presented overwhelming evidence agreeing that orbitals and quantum chemistry are inappropriate for students of chemistry at the level of high school (secondary education). ${ }^{11,12}$ The vague theory confuses the students and the implicit mathematics are too advanced. ${ }^{13}$ Gillespie was especially vehement: "I have been disappointed that localized and hybrid orbitals continue to be taught in introductory courses, and often in high school courses for which they are particularly inappropriate." 14

Young adults must learn in secondary school the essential concepts that can become applicable in the world. "The course must be adapted primarily to the student who will not go farther into the subject. This type of student needs a course that will do two things. He needs first a 'speaking acquaintance' with the common, everyday facts, ideas and nomenclature of the subject in order to become conversationally intelligent regarding it. ... He needs, in the second place, to be able to apply the principles in the solution of every-day problems as they appear....It is the primary purpose of the high-school chemistry course to establish a familiarity with things chemical, and a comprehension of the governing laws and principles." 15

As an analogy, the teaching of Boyle's law is completely acceptable in secondary school but an explanation of gas laws based on statistical mechanics is not. This obvious perception seems less controversial than the quantum debate.

\section{Content of orbitals in first-year university}

In 2011, orbitals gained the imprimatur of the American Chemical Society Examinations Institute (ACS-EI) with their content map of anchoring concepts for general chemistry. ${ }^{16}$ The concepts presented there are designed to span the content that routinely appears in examinations of general chemistry produced by ACS.

The quantum model of the atom is capable of explaining many observations, and it organizes electrons into "orbitals", which are wavefunctions that are identified using quantum numbers. a. The quantum mechanical model of the atom introduces the concept of orbitals, including atomic orbitals.

b. Quantum numbers specify the wavefunctions that are the orbitals. The occupation of atomic orbitals by electrons is summarized in the electron configuration, and this tool is helpful in understanding which atoms form chemical bonds, what type, and how many bonds they form.

a. Electron configuration is a shorthand notation that summarizes the orbital occupations of electrons in an atom or ion.

b. Electron configuration notation for transition-metal ions reflects that they can differ slightly from the patterns observed for main-group ions.

c. Electrons will occupy atomic orbitals following both the aufbau principle and Hund's rule.

Valence-bond theory describes bonds in terms of overlap of electron waves. a. The concept of atomic orbital overlap leading to chemical bonding as embodied in valence-bond theory represents a useful tool for understanding the basic components of the quantum mechanics of bonding.

b. Sigma and pi bonds are a key way to distinguish chemical bonds obtained from valence- bond theory.

A theoretical construct that describes chemical bonding utilizes the construction of molecular orbitals for the bond based on overlap of atomic orbitals on the constituent atoms. 1. Molecular-orbital theory describes chemical bonds via molecular orbitals derived from atomic orbitals.

a. In the quantum model of atoms and molecules, the combination of atomic orbitals leads to the formation of bonding and antibonding molecular orbitals.

b. Bond order can be defined in terms of the occupation of bonding and antibonding orbitals by electrons.

c. Hybrid atomic orbitals are useful in describing bonding, particularly for organic molecules.

This summary creates more questions than it answers. How can the indistinguishability of electrons be rationalized within the concept of an electron configuration when it is impossible to claim that a given electron occupies a specific orbital ${ }^{4}$ Which quantum numbers (within the multiple sets of atomic orbitals, $c f$. Part II) specify a wavefunction? How can one justify the distinctions among molecular orbitals (bonding and antibonding), valence-bond functions (so-called electron waves) and hybrid atomic orbitals (recently effectively debunked $\left.{ }^{17}\right)$ ?

We summarize the use of orbitals in general chemistry in the twenty-first century in four categories:

1) Electron configuration - One set of orbital models for hydrogen serves as a rationalization of a supposed shell structure and subsequently the bonding between atoms of all elements.

2) Periodic table - Atomic orbitals and the associated rules - quantum numbers, Pauli principle, aufbau principle, Hund's rule-lend quantum credence to the organization of the periodic table.

3) Molecular structure - Authors of textbooks seek to justify a structure (such as the angular shape of water) based on the orbitals of the constituent atoms. Part of this structural justification involves the bond order.

4) Reactivity - Some simple reactivity might be illustrated using orbitals to guide the student.

There are other and less generally known uses of orbitals in undergraduate chemical education. For instance, orbitals have been used to describe the shape of isolated, gas-phase atoms: i.e. Mn atoms should be spherical whereas boron should be a prolate spheroid but carbon an oblate spheroid. ${ }^{18}$ Such portrayal is devoid of both experimental and calculational evidence and must be considered untenable.

How important are the uses of orbitals in the four categories mentioned above? Some important previous critiques are specified to grasp the conflict between a complete acceptance of orbitals and their detractors.

1) Electron configuration: Of the electron configurations of gaseous atoms of the elements in their ground states, more than 27 per cent are anomalous in not obeying the combination of the aufbau principle and Hund's rule, ${ }^{19}$ but we continue to show students and to test them on writing a (non-unique ${ }^{4}$ ) electron configuration of an atom and its ions (which have their separate rules) as if there were an absolute pattern to this data. The level of student learning about this topic has been severely criticized: "How many students (or even chemists) ever work with isolated atoms? A few spectroscopists certainly investigate samples in the 
gaseous phase, but nearly all experiments in general chemistry are implemented in aqueous solution. Nearly all industrial chemistry is done in condensed phases; nearly all organic chemistry is done in solution. Why do we waste time and effort teaching beginning students that neutral chromium atoms have $[\mathrm{Ar}] 3 d^{5} 4 s^{1}$ for an electron configuration?"19

2) Periodic table: The original organization of the periodic chart of the chemical elements was based on observations of the recurring similarity of their chemical and physical properties. There is no quantum rationalization of this organization. ${ }^{20}$ Quantum numbers in textbooks are frequently poorly described ${ }^{21}$ students' understanding of quantum numbers, and of the orbital organization of the periodic table, is hence limited..$^{22}$

It is possible to present and to use the periodic table without orbitals and quantum numbers. "What is important for beginning students to understand is that the energies of atomic electrons are measurable. The measured energies indicate that electron energies are quantized. And the periodic table makes it abundantly clear that a primary factor in chemical and physical behavior of the elements is simply the number of the highest energy [purported valence] electrons in their atoms." 12

3) Molecular structure: The atomic orbitals from the elements have been further applied to molecules. Consider a flow chart that we might ask students to follow to provide a three-dimensional structure of a molecule: ${ }^{23}$

"In our current mode of pedagogy we ask our students to follow the path of memorization:

(1) the ground state, gas-phase electron configurations of the atoms;

(2) that electron promotion takes place;

(3) that hybridization of the atomic orbitals occurs;

(4) the shapes of the resulting hybrids;

(5) the tenets of the valence shell electron pair repulsion (VSEPR) model: and

(6) that in trigonal-bipyramidal geometry the lone pairs of electrons go equatorial.

From all this we are now ready to state that, for example, the $\mathrm{SF}_{4}$ molecule adopts a seesaw shape." ${ }^{23}$

This entire process has been criticized. "To use the 'unreality' of atomic electronic configurations (isolated atoms in the gas phase) and to try to create the reality of molecular structure from them, is intellectually suspect. Without an understanding of the mathematics (which I suspect few chemists have), $s p^{3}$ or any other hybridisation label, is just mumbo jumbo. It is simply saying that, if you combine one $s$ orbital with three $p$ orbitals, you get a tetrahedral arrangement of orbitals, leading to bonds which point to the corners of a tetrahedron. Pasteur knew this long before orbitals were thought of!!"24

By far the most common use of orbitals in general chemistry is, however, to bridge the concepts of bonding in molecules from twodimensional Lewis diagrams to three-dimensional structures. The use of electron domains ${ }^{25}$ or a Coulombic mode ${ }^{26}$ has been propounded as an 'orbital-free' model for three-dimensional structure. Jensen described this statement, however, as inaccurate.

"By the 1990s Gillespie was making the further dubious claim that the so-called 'valence-shell electron domain' or VSED model was in fact an alternative to orbital models, rather than a crude method for approximating localized MO, and that the VSEPR approach itself did not require the use of any orbitals whatsoever for its theoretical justification...
However, if it specifically refers to use of the VSED model as an alternative to orbitals, then there is a problem, as the division of the valence-electron density of molecules into spherical, nonoverlapping domains with integral populations consisting of pairs of electrons of opposite spins is identical however you may choose to relabel it - to a use of the Kimball free-cloud model and definitely corresponds, despite claims to the contrary, to the use of an orbital model."27

Bond order is not an observable property; although one can delineate bonds based on a procedure of counting electrons assigned to bonding and antibonding orbitals, it does not greatly add to an understanding of chemistry, especially when bond orders of fractional quantities, resonance structures with varying bond character or hypervalent compounds are discussed. More directly practically, the bond distance and electron density, which are observables, can be used to discuss bonding. The use of computer-generated molecular electrostatic potential (MEP) plots provides a great didactic model to represent bonding: "Many students find this picture [using MEP plots] of multiple bonding less confusing than the standard $\pi$ model.", 28

4) Reactivity: At an introductory level, many educators use orbitals to predict and to explain reactivity, commonly with ancillary concepts such as the octet rule. These explanations are teleological - orbitals and the octet rule are invoked for intentionality or purpose, not for understanding. ${ }^{29}$ These 'hand-waving' explanations are preferred by most chemistry students; even though teleology is an acceptable pedagogic strategy its overuse can affect the learning by students in the long term. The presentation of orbital-caused reactivity to first-year students is a type of "didactical transposition", leading to misconceptions and overgeneralization..$^{30}$

\section{Literature against the teaching of orbitals}

Many chemical educators have clearly deplored the teaching of orbitals until advanced chemistry courses. ${ }^{1,9,23,31,32}$ Gillespie strongly protested the inappropriate nature of quantum mechanics and orbitals in general chemistry. ${ }^{33,34}$ "If the authors of elementary textbooks do not understand quantum mechanics, how can we possibly expect students to understand the subject? All these difficulties could be avoided and a considerable amount of time could be saved if we were to abolish all this material from the curriculum of introductory courses. Quantum mechanics and quantum chemistry should, of course, be studied by all students of chemistry, but not until a later stage in their studies." 35

A knowledge of quantum mechanics, or even its superficial trappings in the form of orbitals, has been proposed to be completely unnecessary for students (the majority) in first-year chemistry who continue with biochemistry or engineering courses. ${ }^{36}$ Despite these carefully researched recommendations, at least one textbook of general chemistry for the latter students ${ }^{37}$ devotes a proportion, $~ 16 \%$ - similar to that in other textbooks, of its extent to orbitals. One author labeled quantum mechanics as "Unteachable at the General Level". ${ }^{8}$ Pauling wrote on several occasions about general chemistry without molecular orbitals (although he undoubtedly preferred valence-bond theory). ${ }^{1,39}$

We leave the last words in this section to Gillespie: "Concepts such as hybrid and molecular orbitals and the equations of thermodynamics are too abstract and too difficult for an introductory course. Moreover, they are unnecessary... at best students acquire only a very superficial understanding that often involves misconceptions that need to be unlearned if the student continues in further chemistry courses. And at worst students just memorize the appropriate jargon needed 
to pass tests and examinations and they understand and remember almost nothing." 33

\section{Literature defense of the teaching of orbitals}

In a spirit of objective discussion, we present some arguments for the use of orbitals, and their critique.

In 1988 Edmiston $^{40}$ refuted the arguments of Sanderson that chemistry can be taught without orbitals. ${ }^{31}$ Edmiston provided several items in this refutation: orbitals give students insights into many properties of molecules; molecular-orbital calculations ab initio (and their visualization) are available; chemical bonding is better understood with orbitals; the reasons that molecules have their shapes can be rationalized. He recommended that localized molecular orbitals (LMO) corresponding to bonds, lone pairs and inner shells - despite that electrons are absolutely indistinguishable - be shown to students and then form the delocalized spectroscopic MO as simple linear combinations of these LMO, and that what is needed is an improved presentation. Molecular binding energies, especially in contrast to the method of Sanderson, are properties that he emphasized to be best explained in MO theory. "Binding effects result from the positive overlap of atomic orbitals to form bonding LMO, and antibonding effects result from negative overlap, causing MO nodes in 'bond regions'. To ignore the teaching of these most basic ideas would deprive students of much important understanding." ${ }^{40}$

This understanding might be important for physical chemistry, but not general chemistry; one must resist the conversion of general chemistry into "baby p-chem". ${ }^{9}$ The use of quantum-chemistry software (molecular-orbital calculations so-called $a b$ initio) to determine molecular properties has unquestionably altered the way that many chemists conduct their research. During the twenty-first century, it will be simply impossible to include all this information within a curriculum for undergraduates.

Schaefer defended the presentation of quantum mechanics in general chemistry by proclaiming "Quantum mechanics is arguably the most significant intellectual achievement of the twentieth century. Furthermore, quantum mechanics has been foundational to why chemistry is understood in a much more profound manner than was the case in 1926. Whether one likes it or not, orbitals have become the lingua franca of chemistry since the 1963 paper of Woodward and Hoffmann." ${ }^{41}$

Here, orbitals are clearly confused with quantum mechanics: orbitals are merely artefacts of wave mechanics, which is in turn only one method among many that collectively constitute quantum mechanics. ${ }^{42}$ One can acknowledge the great achievement of quantum mechanics while seeking alternative approaches for chemical education in the twenty-first century. Few students of general chemistry will learn, and even fewer will ever use, the WoodwardHoffman rules.

These articles seem to be the only defense of the inclusion of orbitals in general chemistry. As indicated in the introduction, in the twenty-first century all textbooks include the material and most modern instructors do not criticize this choice.

\section{Literature in research in chemical education}

Instead of discussing the necessity of orbitals, several authors have published articles in the twenty-first century lamenting the difficulty of teaching quantum mechanics and orbitals and the (understandable) confusion that students show when tested on quantum-mechanical principles.

What do first-year undergraduate students actually learn about quantum mechanics and orbitals? The research indicates 'not much'.
Tsaparlis wrote that Greek undergraduate students in chemistry lack a clear understanding of the concepts of atomic and molecular orbitals. ${ }^{43}$ Nakiboglu noted that Turkish undergraduates that proceed to become teachers of chemistry in secondary school show serious misconceptions about orbitals. ${ }^{44}$ Conceicao used computer software to alleviate partially the problems that USA students have with the concept of orbitals. ${ }^{45}$ Taber noted that further research is required to eliminate the misconceptions of students about orbitals at Cambridge. ${ }^{46}$ Chamizo et al. complained that ideas such as orbitals are unconnected to their utility or practical use and that they are presented to Mexican students with no experimental evidence (because there is none!). ${ }^{47}$ Bouayad et al. emphasized the confusion of Moroccan students and directly identified quantum mechanics as an impediment to deep understanding. ${ }^{48}$ Lima and Silva observed the lack of clarity and knowledge of quantum matters in Brazilian students. ${ }^{49}$ Internationally, students have the same difficulties; internationally, orbitals are the problem!

\section{CONCLUSIONS}

Part I here provides a summary of the great debate about the definition, use and misuse of orbitals in general chemistry. Despite the inherent sense in a definition of orbital by Brown et al., ${ }^{2}$ the teaching of general chemistry involves a mixture of chemical species and properties, on the one hand, and algebraic formulae, on the other hand, which belong to disparate logical classes. ${ }^{50}$ Their admixture creates universal confusion unworthy of a scientific discipline. ${ }^{51}$

The question remains whether orbitals comprise an essential and unique model for teaching general chemistry. The four main uses of orbitals in general chemistry - electron configuration, periodic table, molecular structure and simple reactivity - are shown above to be less essential than most textbooks state. In Part II we demonstrate that, even within wave mechanics, there are multiple schemes of orbitals, which further undermine the application of any one scheme for teaching. In Part III we proceed to combine the information in Parts I and II to present modern guidelines for the teaching of general chemistry, and to propose a course curriculum without the use of orbitals.

\section{ACKNOWLEDGEMENTS}

El Centro de Investigaciones en Productos Naturales (CIPRONA) and la Escuela de Química, Universidad de Costa Rica (UCR) provided support. We thank several students and professors at UCR for helpful discussion.

\section{REFERENCES}

1. Quotation in Bent, H. A.; J. Chem. Educ. 1984, 61, 421.

2. Brown, T. L.; LeMay, H. E.; Brusten, B. E.; Murphy, C. J.; Woodward, P. M.; Chemistry: the Central Science, 12 ${ }^{\text {th }}$ ed., Pearson: Boston, 2012.

3. Mulliken, R. S.; Science 1967, 157, 13.

4. Truhlar, D. G.; Hiberty, P. C.; Shaik, S.; Gordon, M. S.; Danovich, D.; Angew. Chem. Int. Ed. 2019, 58, 12332.

5. Dauth, M.; Wiessner, M.; Feyer, V.; Schöll, A.; Puschnig, P.; Reinert, F.; Kümmel, S.; New J. Phys. 2014, 16, 103005.

6. Taber, K. S.; Chem. Educ. Res. Pract. 2002, 3, 145; Taber, K. S.; Chem. Educ. Res. Pract. 2002, 3, 159.

7. Sánchez-Gómez, P. J.; Martín, F.; Chem. Educ. Res. Pract. 2003, 4, 131.

8. Taber, K. S.; Sci. Educ. 2005, 89, 94.

9. Bell, J. A. In Sputnik to Smartphones: A Half-Century of Chemistry Education; Orna. M. V., ed.; ACS Publications: Washington, DC, 2015, pp. 25-43. 
10. Morwick, J. J.; J. Chem. Educ. 1979, 56, 262.

11. Gillespie, R. J.; Humphreys, D. A.; J. Chem. Educ. 1980, 57, 348; Berry, K. O.; J. Chem. Educ. 1986, 63, 697; Gold, M.; J. Chem. Educ. 1988, 65, 780; Shiland, T. W.; J. Chem. Educ. 1995, 72, 215; Cervellati, R.; Perugini, D.; J. Chem. Educ. 1981, 58, 568; Tsaparlis, G.; Pappa, E. T.; Byers, B.; Chem. Teacher Int. 2019, 2, 20190002.

12. Ruis, S. P.; J. Chem. Educ. 1988, 65, 720.

13. Tsaparlis, G.; Papahotis, G.; Chem. Educ. Res. Pract. 2002, 3, 129; Tsaparlis, G.; Papaphotis, G.; Int. J. Sci. Educ. 2009, 31, 895.

14. Cardellini, L.; J. Chem. Educ. 2010, 87, 482.

15. Bayles, E. E.; J. Chem. Educ. 1930, 7, 1317.

16. Holme, T.; Murphy, K.; J. Chem. Educ. 2012, 89, 721.

17. Lamoureux, G.; Ogilvie, J. F.; Quim. Nova 2019, 42, 812.; Lamoureux, G.; Ogilvie, J. F.; Quim. Nova 2019, 42, 817.

18. Johnson, R. C.; Rettew, R. R.; J. Chem. Educ. 1965, 42, 145.

19. Millikan, R. C.; J. Chem. Educ. 1982, 59, 757.

20. Ostrovsky, V. N.; Found. Chem. 2005, 7, 235; Schwarz, W. H. E.; Found. Chem. 2007, 9, 139.

21. Niaz, M., Fernandez, R.; Int. J. Sci. Educ. 2008, 30, 869.

22. Temel, S.; Ozcan, O.; SHS Web of Conferences 2018, 48, 1002.

23. Zuckerman, J. J.; J. Chem. Educ. 1986, 63, 829.

24. Johnstone, A. H.; Univ. Chem. Educ. 2000, 4, 34.

25. Gillespie, R.; Robinson, E. A.; Angew. Chem., Int. Ed. 1996, 35, 495.

26. Sacks, L. J.; J. Chem. Educ. 1986, 63, 288; Sacks, L. J.; J. Chem. Educ. 1986, 63, 373; Sacks, L. J.; J. Chem. Educ. 1986, 63, 48.

27. Jensen, W. B.; J. Chem. Educ. 2014, 91, 1106.

28. Shusterman, A. J.; Shusterman, G. P.; J. Chem. Educ. 1997, 74, 771.

29. Talanquer, V.; J. Chem. Educ. 2013, 90, 1419.

30. Talanquer, V.; Int. J. Sci. Educ. 2007, 29, 853.

31. Sanderson, R. T.; J. Chem. Educ. 1986, 63, 845.

32. Ogilvie, J. F.; J. Chem. Educ. 1990, 67, 280; Tsaparlis, G. J. Chem. Educ. 1997, 74, 922; Pritchard, H. O. J. Chem. Educ. 2012, 89, 301.
33. Gillespie, R. J.; J. Chem. Educ. 1991, 68, 192.

34. Gillespie, R. J.; J. Chem. Educ. 1996, 73, 622.

35. Gillespie, R. J.; Chemistry in Canada 1976, 12, 23.

36. Hawkes, S. J.; J. Chem. Educ. 1992, 69, 178; Hawkes, S. J. J. Chem. Educ. 2000, 77, 321.

37. Brown, L. S.; Holme, T.; Chemistry for Engineering Students, $3^{\text {rd }}$ ed., Centage Learning: Stamford, 2015.

38. Spencer, J. N.; J. Chem. Educ. 1992, 69, 182.

39. Pauling, L.; J. Chem. Educ. 1980, 57, 38; Pauling, L. J. Chem. Educ. 1992, 69, 519.

40. Edmiston, C. K.; J. Chem. Educ. 1988, 65, 219.

41. Schaefer, H. F.; J. Chem. Educ. 1993, 70, 782.

42. Styer, D. F.; Balkin, M. S.; Becker, K. M.; Burns, M. R.; Dudley, C. E.; Forth, S. T.; Gaumer, J. S.; Kramer, M. A.; Oertel, D. A.; Park, L. H.; Rinkoski, M. T.; Smith, C. T.; Wotherspoon, T. D.; Am. J. Phys. 2002, 70, 288.

43. Tsaparlis, G.; Res. Sci. Educ. 1997, 27, 271; Stefani, C.; Tsaparlis, G.; J. Res. Sci. Teach. 2009, 46, 520

44. Nakiboglu, C.; Chem. Educ. Res. Pract. 2003, 4, 443.

45. Conceicao, J.; Koscinski, J. T.; Chem. Educ. 2003, 8, 378.

46. Taber, K. S.; Sci. Educ. 2005, 89, 94.

47. Martinez, A.; Valdés, J.; Talanquer, V.; Chamizo, J. A.; Educ. Quim. 2012, 23, 361.

48. Bouayad, A.; Kaddari, F.; Lachkar, M.; Elachqar, A.; Procedia - Social and Behavioral Sciences 2014, 116, 4612.

49. Lima, M. M.; Silva, D. P. B.; Sci. Nat. 2019, 1, 10.

50. Primas, H.; Quantum Chemistry and Reductionism: Lecture Notes in Chemistry Vol. 24. Springer-Verlag: Berlin, 1981.

51. Zoller, U.; J. Res. Sci. Teach. 1990, 27, 1053. 